\title{
THE BLOCK GAUSS-SEIDEL METHOD IN SOUND TRANSMISSION PROBLEMS
}

\author{
J. Poblet-Puig, A. Rodríguez-Ferran ${ }^{\dagger}$ \\ Laboratori de Càlcul Numèric \\ E.T.S. d'Enginyers de Camins, Canals i Ports de Barcelona \\ Universitat Politècnica de Catalunya
}

May 17, 2011

\begin{abstract}
Sound transmission through partitions can be modelled as an acoustic fluidelastic structure interaction problem. The block Gauss-Seidel iterative method is used in order to solve the finite element linear system of equations. The blocks are defined, respecting the fluid and structural domains. The convergence criterion is analysed and interpreted in physical terms by means of simple onedimensional problems. This analysis highlights the negative influence on the convergence of a strong degree of coupling between the acoustic domains and the structure. A selective coupling strategy has been developed and applied to problems with strong coupling (e.g. double walls).
\end{abstract}

\section{Introduction}

Fluid-structure interaction is the key aspect of many acoustic problems of practical interest. This is the case, for instance, of sound propagation through partitions in buildings. In this field, vibroacoustic equations are solved in the frequency domain and multiple frequencies have to be considered in order to obtain the frequency response spectrum of the system.

Several formulations of the vibroacoustic equations are available in the literature [1-3]. The structure is usually described by means of the displacement formulation while several options have been used for the fluid. When the fluid is described by means of displacement or a mixed pressure-displacement formulations the coupled problem is globally symmetric. However there exist spurious modes. Modifications

*correspondence: UPC, Campus Nord B1, Jordi Girona 1, E-08034 Barcelona, Spain, e-mail: jordi.poblet@upc.edu

†e-mail: antonio.rodriguez-ferran@upc.edu 
have to be introduced in order to avoid this drawback, see for example [4]. This is not the case of the velocity potential [3] or pressure formulations [5-7]. In the first case the systems of equations to be solved are symmetrical but an extra postprocessing is required if the final output is the acoustic pressure (which is often the case in building acoustics). In the pressure formulation the systems of equations are unsymmetrical but smaller than in displacement or mixed formulations.

In all of these cases the finite element discretisation leads to systems of linear equations with a block structure. The diagonal blocks in the global matrix are typically symmetric and indefinite, but the off-diagonal blocks (which represent the coupling between the acoustic fluid and the elastic structure) break the symmetry of the global matrix.

For this reason, a monolithic solution approach requires the use of general solvers for unsymmetrical and indefinite matrices, such as Crout factorisation or GMRES iterations with an appropriate preconditioner $[8,9]$. Alternatively, block iterative solvers can be used. By doing so, the symmetry of the diagonal blocks can be exploited, and the storage requirements are decreased.

The block Gauss-Seidel iterative solver is considered here. The well-known convergence condition (spectral radius of iteration matrix smaller than one) is interpreted from a physical viewpoint, by considered simple, one-dimensional vibroacoustic models. This analysis shows the detrimental effect on the convergence of the iterative solver of $i$ ) the excitation frequency being close to an acoustic or structural eigenfrequency and ii) a large level of coupling between the acoustic fluid and the structure.

The coupling in a fluid-structure problem can be weak or strong depending on different factors such as the physical properties of the media, the geometry of the structure or the imposed boundary conditions. This has important consequences in the performance of the solving strategies and has been widely studied when the problem is solved in the time domain (see for example [10-14]). Computational costs can be drastically reduced if the coupling is weak and the fluid and structure problems can be solved in a staggered way. In the frequency domain, it is not easy to predict when the coupling is strong or weak. In the first case a monolithic solver is required while in the second case a one-way coupling strategy is enough. The block GaussSeidel method considered here is between these two strategies, taking advantage of weak coupling when it is possible and reducing computational costs.

The block Gauss-Seidel method can be understood as a domain decomposition method. These methods base their efficiency in the splitting of the physical domain of the problem into smaller subdomains. The system of equations is then solved at two different levels. On the one hand each subdomain is solved as an individual problem and on the other hand the continuity between them is imposed. These techniques have been mainly designed to be used in parallel computing machines. Each CPU deals with a single smaller domain using the more adequate solver for each region.

In [15-17] domain decomposition techniques have been used in order to solve scattering problems governed by the Helmholtz equations in big physical domains. The continuity of the pressure field (and its normal derivative) in the interface between regions is imposed by means of Lagrange multipliers. Moreover each subdomain has to be regularised by means of fictitious boundary conditions in order to avoid problems caused by artificial eigenfrequencies. Domain decomposition techniques has also 
been used for vibroacoustic problems. In [18] the partitions have been done in both the acoustic domains and the structure. Finally, in [19-21], block Jacobi and block Gauss-Seidel algorithms have been used for vibroacoustic problems where the decomposition of the domain strictly respects the physical regions (fluid and structure). The only interface between subdomains is the fluid-structure boundary. Since the goal in [19-21] is to propose a general solver (also for strongly coupled problems) their discussion is focused on the convergence of the methods. It seems clear that using the physical interface conditions in order to transfer information between fluid and structural subdomains leads to divergence in a large number of situations. They propose relaxed coupling conditions that cause the block Gauss-Seidel algorithm to have fast convergence for all the analysed situations. In the application examples the performance of the modified algorithms around the eigenfrequencies of the problem has not been analysed. Moreover, the use of the modified interface conditions require some modifications at finite element level.

An outline of the paper follows. The convergence condition and its physical interpretation are covered in Section 3. The application examples of Section 4 corroborate this interpretation, and motivate the selective coupling strategy presented in Section 5, which is applied to the problem of sound propagation through double walls. The concluding remarks of Section 6 close the paper.

\section{The block Gauss-Seidel algorithm}

The block Gauss-Seidel algorithm will be presented in matrix form. The coupled system of linear equations is

$$
\left[\begin{array}{cc}
\mathbf{F} & \mathbf{C}_{F S} \\
\mathbf{C}_{S F} & \mathbf{S}
\end{array}\right]\left\{\begin{array}{l}
\mathbf{x}_{F} \\
\mathbf{x}_{S}
\end{array}\right\}=\left\{\begin{array}{l}
\mathbf{f}_{F} \\
\mathbf{f}_{S}
\end{array}\right\}
$$

The pressure-displacement formulation is taken as reference here. $\mathbf{F}$ is the flexibility matrix governing the fluid domain with nodal unknowns $\mathbf{x}_{F}$ (typically pressures) and $\mathbf{S}$ is the stiffness matrix governing the structural domain with nodal unknowns $\mathbf{x}_{S}$ (typically displacements and rotations). If FEM is used, $\mathbf{F}$ and $\mathbf{S}$ are typically sparse, symmetric and indefinite matrices. $\mathbf{f}_{F}$ and $\mathbf{f}_{S}$ are the forces acting in the fluid and structural domains. The coupling is taken into account by means of matrices $\mathbf{C}_{F S}$ and $\mathbf{C}_{S F}$. The forces acting on the structure due to the acoustic pressures in the fluid are

$$
\mathbf{f}_{S F}=\mathbf{C}_{S F} \mathbf{x}_{F}
$$

and the acoustic forces in the fluid contour caused by the structural vibrations are

$$
\mathbf{f}_{F S}=\mathbf{C}_{F S} \mathbf{x}_{S}
$$

The global matrix of Equation (1) is non-symmetric for the more widely used formulations. The block Gauss-Seidel algorithm is summarised in Table 1.

The initial guess can be chosen as the solution of the uncoupled problems. The convergence is checked by means of the relative errors in the solution

$$
e_{F}^{(i)}=\frac{\left\|\mathbf{x}_{F}^{(i)}-\mathbf{x}_{F}^{(i+1)}\right\|}{\left\|\mathbf{x}_{F}^{(i+1)}\right\|} ; \quad e_{S}^{(i)}=\frac{\left\|\mathbf{x}_{S}^{(i)}-\mathbf{x}_{S}^{(i+1)}\right\|}{\left\|\mathbf{x}_{S}^{(i+1)}\right\|}
$$


Table 1: The block Gauss-Seidel method

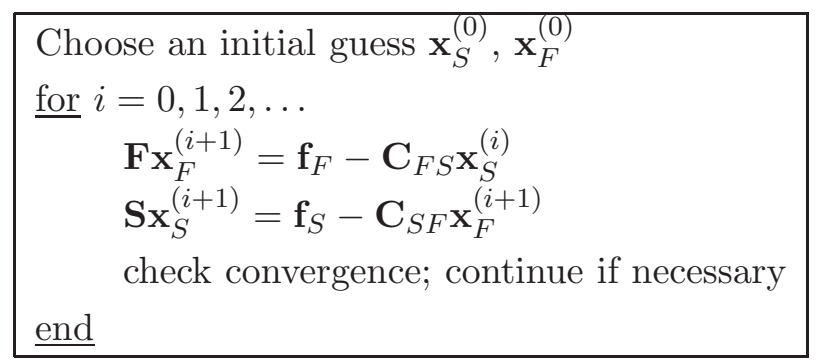

and the relative residual

$$
r_{F}^{(i)}=\frac{\left\|\mathbf{F} \mathbf{x}_{F}^{(i)}+\mathbf{C}_{F S} \mathbf{x}_{S}^{(i)}-\mathbf{f}_{F}\right\|}{\left\|\mathbf{f}_{F}\right\|}
$$

The two systems of equations in Table 1 have to be solved several times with different force vectors but constant matrices $\mathbf{F}$ and $\mathbf{S}$. This has to be exploited for maximum efficiency. A first option is to use a direct solver (for small matrix dimensions) and save the factorisation of the matrices. Another possibility is to use the adequate iterative solver (GMRES, MINRES,... see [8,9] for more details and [22-24] for robust implementations) and save the preconditioner, which is calculated only once for $i=0$ and can be reused for the successive iterations. Wave problems often require to perform calculations for successive frequencies or different types of force terms. Matrices are then very similar. The possibility of using the same preconditioner for several successive frequencies has also to be considered.

\section{Analysis of the block Gauss-Seidel method}

\subsection{The convergence condition}

As other stationary iterative methods, the block Gauss-Seidel algorithm converges if the spectral radius $\rho$ (i.e. the maximum modulus of the eigenvalues) of the iteration matrix $\mathbf{G}$ is less than one, see [9]. The algorithm in Table 1 can be rewritten as

$$
\left\{\begin{array}{c}
\mathbf{x}_{F}^{(i+1)} \\
\mathbf{x}_{S}^{(i+1)}
\end{array}\right\}=\left[\begin{array}{cc}
\mathbf{0} & -\mathbf{F}^{-1} \mathbf{C}_{F S} \\
\mathbf{0} & \mathbf{S}^{-1} \mathbf{C}_{S F} \mathbf{F}^{-1} \mathbf{C}_{F S}
\end{array}\right]\left\{\begin{array}{c}
\mathbf{x}_{F}^{(i)} \\
\mathbf{x}_{S}^{(i)}
\end{array}\right\}+\left\{\begin{array}{c}
\mathbf{F}^{-1} \mathbf{f}_{F} \\
\mathbf{S}^{-1}\left(\mathbf{f}_{S}-\mathbf{C}_{S F} \mathbf{F}^{-1} \mathbf{f}_{F}\right)
\end{array}\right\}
$$

The iteration matrix $\mathbf{G}$ is the matrix in Equation (6), so the convergence condition is

$$
\rho\left(\mathbf{S}^{-1} \mathbf{C}_{S F} \mathbf{F}^{-1} \mathbf{C}_{F S}\right)<1
$$

\subsection{Physical interpretation of the convergence condition}

The simplified model of Figure 1 will be used in order to understand and illustrate the phenomena of vibroacoustic coupling and the performance of the block GaussSeidel algorithm. A vibrating mass is coupled with an acoustic domain. Both can be 


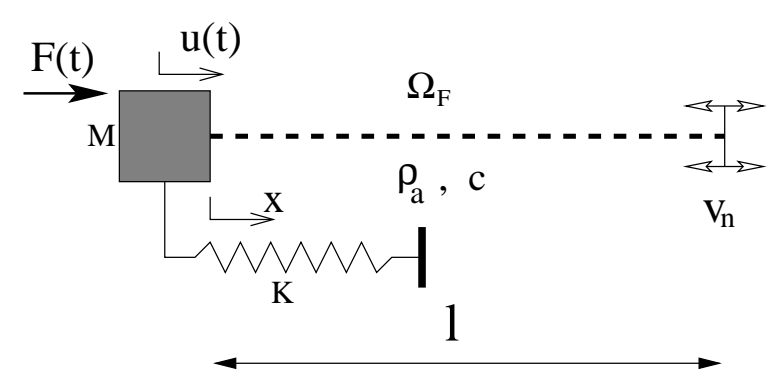

Figure 1: Simple one-dimensional coupled system with two degrees of freedom.

excited: the mass by means of an exterior force $F(t)=\operatorname{Re}\left\{\varphi e^{\mathrm{i} \omega t}\right\}$ and the acoustic fluid cavity by an exterior imposed velocity $v_{\boldsymbol{n}}$. Note that the model is formulated for a unit surface. Thereby $\varphi$ is the phasor of force per unit surface, and $M$ and $K$ the mass and stiffness per unit surface.

The interaction between the acoustic fluid and the single mass can be characterised by the pressure applied by the fluid on the mass and the displacement imposed by the mass at the acoustic contour.

The governing equation and boundary conditions for the fluid domain are

$$
\begin{array}{rlr}
\frac{d^{2} p(x)}{d x^{2}}+k^{2} p(x) & =0 & x \text { in } \Omega_{F} \\
\left.\frac{d p(x)}{d x}\right|_{x=0} & =\rho_{F} \omega^{2} u & \\
\left.\frac{d p(x)}{d x}\right|_{x=\ell} & =-\rho_{F} \mathrm{i} \omega v_{n} &
\end{array}
$$

and if the frequency of the problem is a real value, the pressure field is

$$
p(x)=C_{1} \cos (k x)+C_{2} \sin (k x)
$$

where $C_{1}$ and $C_{2}$ are unknown complex constants. Taking into account the dynamic equilibrium of the single mass, a linear system with three equations results:

$$
\left[\begin{array}{cc|c}
\sin (k \ell) & -\cos (k \ell) & 0 \\
0 & 1 & -\rho_{F} \omega c \\
\hline 1 & 0 & K-\omega^{2} M
\end{array}\right]\left\{\begin{array}{c}
C_{1} \\
C_{2} \\
\hline u
\end{array}\right\}=\left\{\begin{array}{c}
\rho_{F} \mathrm{i} c v_{\boldsymbol{n}} \\
0 \\
\hline \varphi
\end{array}\right\}
$$

This system is the particularisation for this simple one-dimensional example of Equation (1). The convergence condition (7) leads to

$$
\rho(\mathbf{G})=\frac{\cos (k \ell) \rho_{F} \omega c}{\sin (k \ell)\left(K-\omega^{2} M\right)}<1
$$

A similar analysis can be done for the one-dimensional model with two fluid domains shown in Figure 2 and [25], which represents two rooms separated by a partition. The expression of the spectral radius is

$$
\rho(\mathbf{G})=\frac{\rho_{F} \omega c}{K-\omega^{2} M}\left(\frac{\cos \left(k \ell_{1}\right)}{\sin \left(k \ell_{1}\right)}+\frac{\cos \left(k \ell_{2}\right)}{\sin \left(k \ell_{2}\right)}\right)
$$




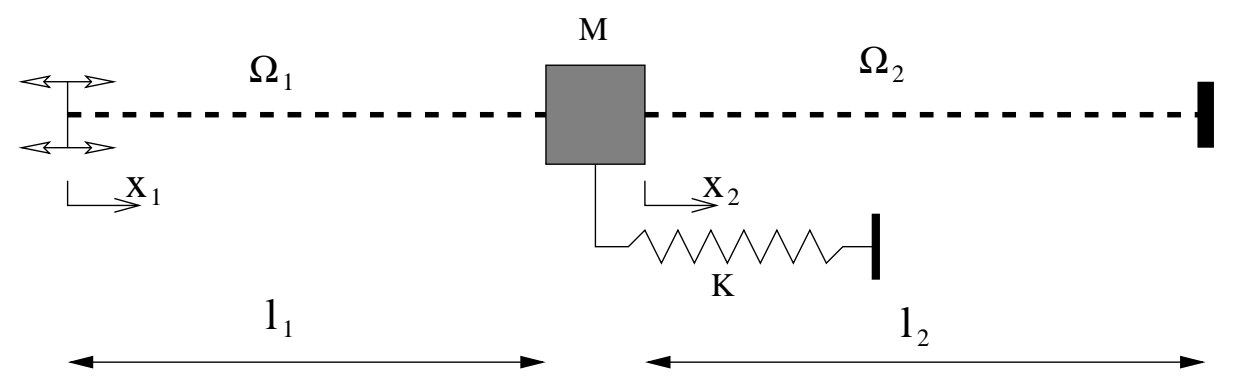

Figure 2: Simple one-dimensional coupled system with two acoustic domains.

Several conclusions can be obtained from Equations (13) and (14). The method is less efficient for denser fluids (i.e. larger density $\rho_{F}$ ) or fluids with higher wave speed $c$, because the coupling between the structure and the fluid increases.

The geometry of the problem is also important. For this one-dimensional case, the geometry is represented by terms $\cos (k \ell)$ and $\sin (k \ell)\left(\ell=\ell_{1}\right.$ or $\ell_{2}$ in Equation (14)). If $\sin (k \ell) \approx 0$ the method will not converge. This happens for the eigenfrequencies of the acoustic cavity, $k_{n}=n \pi / \ell$, but also when $\ell$ is very small (small fluid domains). If $\cos (k \ell)=0$ the method converges in one iteration. This is a very specific situation of the one-dimensional model and cannot be generalised to higher dimensions.

Note that the method also diverges for frequencies close to the structural eigenfrequency $\sqrt{K / M}$. Finally, Equations (13) and (14) also show that the performance of the iterative solver improves with the frequency.

A similar parameter $\left(\lambda=\rho_{F} c / \rho_{S} t \omega\right)$ has been defined by [26]. $\mathrm{t}$ is the typical thickness of the structure and $\rho_{S}$ its density. However, $\lambda$ does not take into account the influence of the geometry nor the stiffness.

By condensing out the unknown $C_{2}$ and noting from Equation (11) that $C_{1}$ is $p(x=0)$, system (12) can be recast as

$$
\left[\begin{array}{cc}
\sin (k \ell) & -\rho_{F} \omega c \cos (k \ell) \\
1 & K-\omega^{2} M
\end{array}\right]\left\{\begin{array}{c}
p(x=0) \\
u
\end{array}\right\}=\left\{\begin{array}{c}
\rho_{F} \mathrm{i} c v_{\boldsymbol{n}} \\
\varphi
\end{array}\right\}
$$

and $\rho(\mathbf{G})$ can then be viewed as the ratio of stiffness of the fluid and the structure, including the effect of coupling:

$$
\rho(\mathbf{G})=\frac{(d p(x=0) / d u)_{F}}{(d p(x=0) / d u)_{S}}
$$

The subscript $F(S)$ means here derivative from the point of view of the fluid (structure).

The conceptual behaviour of the fluid-structure system has been plotted in Figure 3. The harmonic equilibrium is reached at the pair $\mathbf{x}_{S}^{*}-\mathbf{x}_{F}^{*}$. The acoustic pressure caused by the structural displacement is

$$
\mathbf{x}_{F S}=-\mathbf{F}^{-1} \mathbf{C}_{F S} \mathbf{x}_{S}^{*}
$$

and the displacement caused by the pressure is

$$
\mathbf{x}_{S F}=-\mathbf{S}^{-1} \mathbf{C}_{S F} \mathbf{x}_{F}^{*}
$$




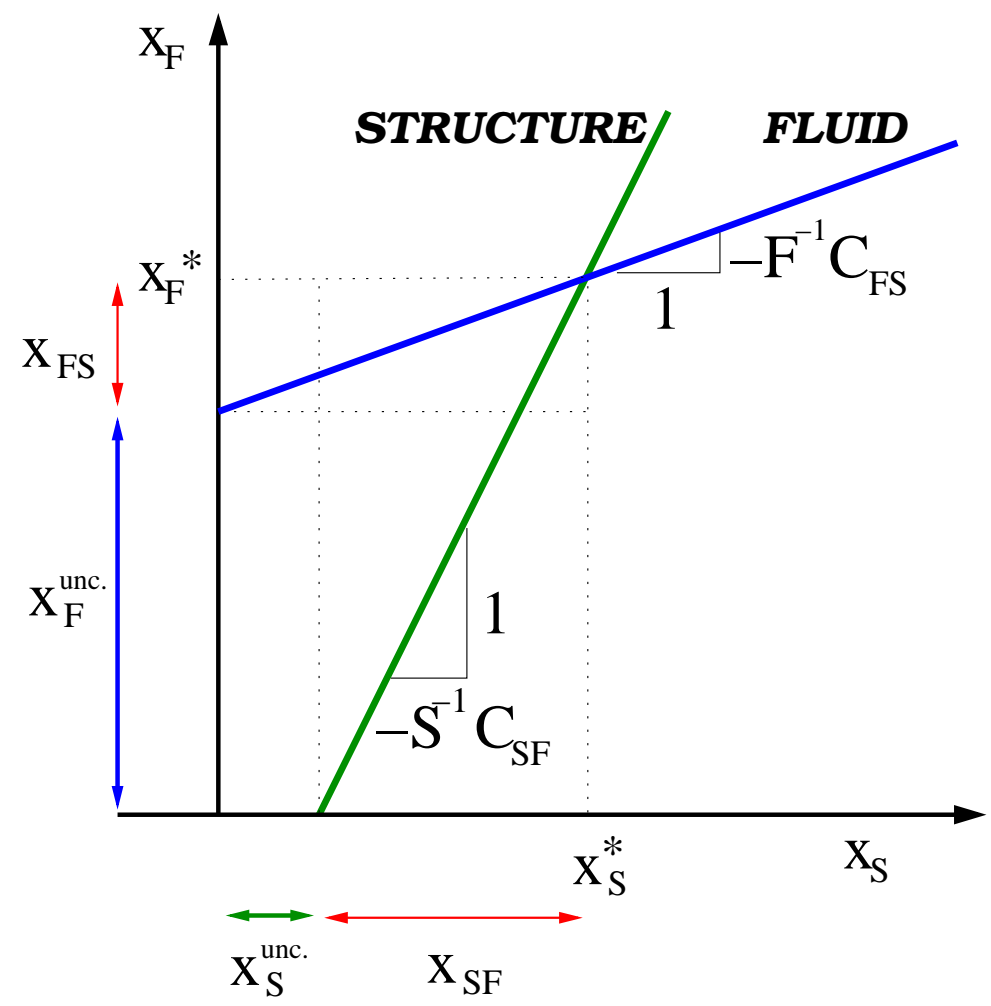

Figure 3: Conceptual behaviour of a coupled fluid-structure system.

Figure 4 shows a sketch of the convergence and divergence of the algorithm depending on the spectral radius.

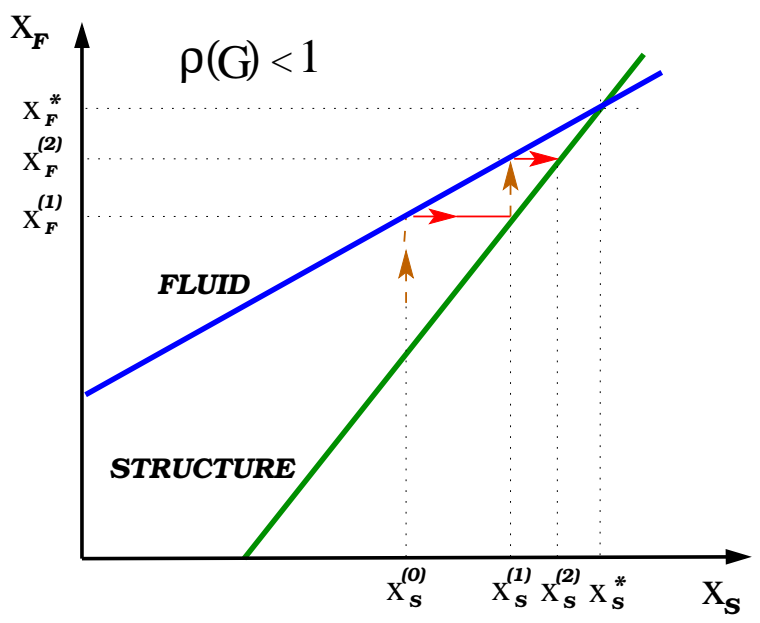

(a)

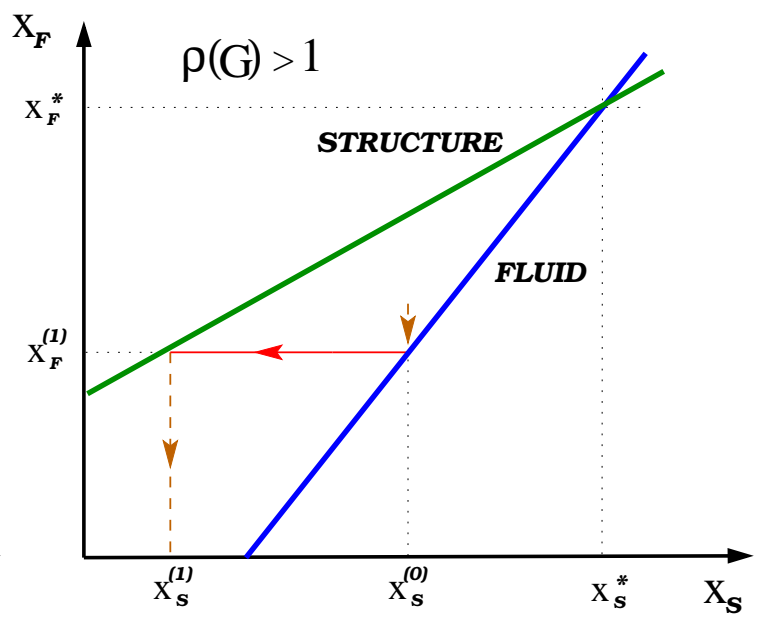

(b)

Figure 4: Convergence of the block Gauss-Seidel algorithm: (a) Convergence for $\rho(\mathbf{G})<1$; (b) Divergence for $\rho(\mathbf{G})>1$. 


\section{Application examples}

The performance of the block Gauss-Seidel method is illustrated here with various $2 \mathrm{D}$ and $3 \mathrm{D}$ vibroacoustic problems (sound transmission through a single wall). The method has already been used to study the sound transmission through single and double walls in $[25,27]$. The goals are 1) to show the influence on the convergence of the iterative solver of $i$ ) the damping, ii) the coupling between fluid and structure and iii) acoustic and structural eigenfrequencies, and 2) to demonstrate the use of the solver in practical simulations. A FEM-FEM approach (i.e. finite elements for the fluid and acoustic domains) is used here.

\subsection{Influence of damping}

Two acoustic domains are separated by a single wall (represented in this two-dimensional setting by a concrete beam), see Figure 5 . The acoustic excitation is a punctual sound source placed in the left bottom corner of the first domain, at a distance of $0.5 \mathrm{~m}$ to the contours. The room dimensions are $3 \times 3 \mathrm{~m}^{2}$ and $4 \times 3 \mathrm{~m}^{2}$. The material and geometrical parameters are summarised in Table 2. Note that we are dealing with air, which is a very light fluid. This is the typical situation where the method will have a very good behaviour. A relative tolerance of $10^{-9}$ is used in the stopping criteria defined in Equations (4) and (5).

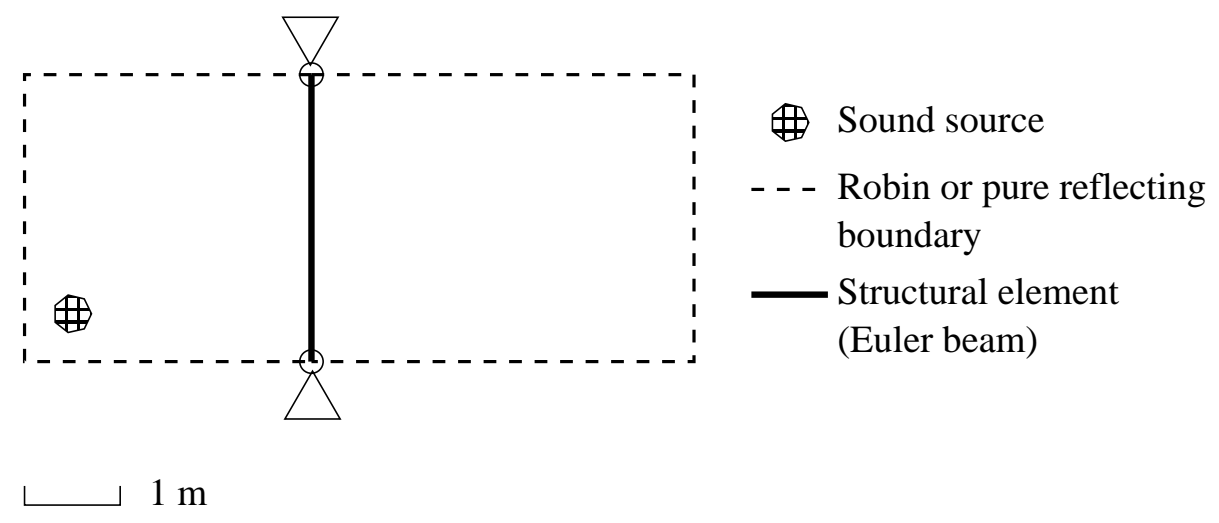

Figure 5: Sound transmission through a single wall

Two different situations have been analysed. On the one hand, an undamped problem (no acoustic absorption and no structural damping). On the other hand, the same problem with an acoustic absorption of $30 \%$ at the boundaries (introduced by means of a Robin boundary condition) and hysteretic structural damping (5\%). These are reasonable values.

The results (number of iterations required) have been plotted in Figure 6. Note that damping considerably decreases the number of iterations required, especially near eigenfrequencies. 
Table 2: Material and geometrical data for the acoustic domains (air), the heavy single wall and the lightweight leaves of the double wall.

\begin{tabular}{llll}
\hline & \multicolumn{2}{c}{ STRUCTURE } \\
Meaning & Symbol & Heavy & Lightweight \\
\hline Young's modulus & $E$ & $2.943 \cdot 10^{10} \mathrm{~N} / \mathrm{m}^{2}$ & $4.5 \cdot 10^{9} \mathrm{~N} / \mathrm{m}^{2}$ \\
Poisson's ratio & $\nu$ & 0.25 & 0.25 \\
Wall density & $\rho_{S}$ & $2500 \mathrm{~kg} / \mathrm{m}^{3}$ & $913 \mathrm{~kg} / \mathrm{m}^{3}$ \\
Wall thickness & $\mathrm{t}$ & $0.10 \mathrm{~m}$ & $0.013 \mathrm{~m}$ \\
Loss factor & $\eta$ & $0-5 \%$ & $0-5 \%$ \\
\hline
\end{tabular}

\begin{tabular}{lll}
\hline & FLUID & \\
Meaning & Symbol & Value \\
\hline Speed of sound & $\mathrm{c}$ & $340 \mathrm{~m} / \mathrm{s}$ \\
Density of fluid & $\rho_{F}$ & $1.18 \mathrm{~kg} / \mathrm{m}^{3}$ \\
Source strength & $Q$ & $0.005 \mathrm{i} \mathrm{m} / \mathrm{s}$ \\
Acoustic absorption & $\alpha$ & $0-30 \%$ \\
\hline
\end{tabular}

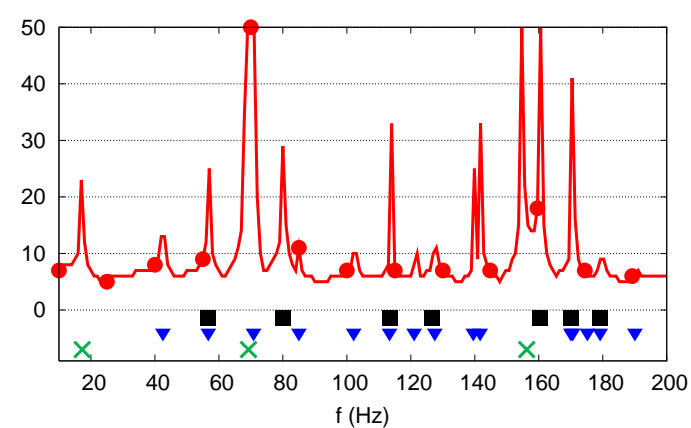

Iterations
Eig. $\Omega_{\mathrm{F}}{ }^{(1)}$

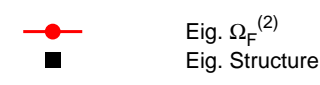

(a)
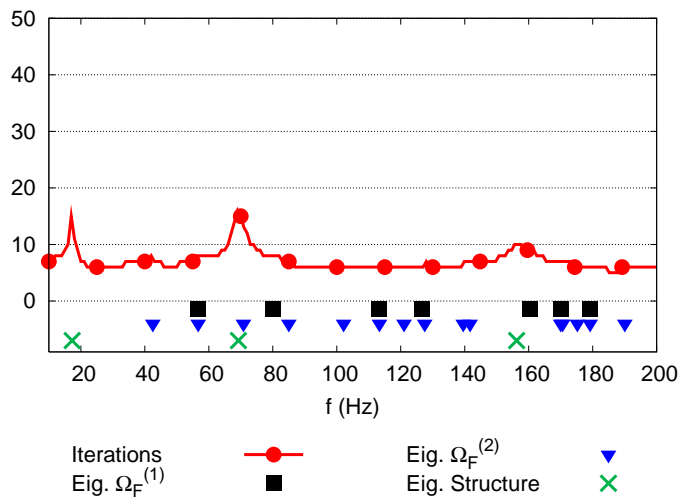

(b)

Figure 6: Iterations of the block Gauss-Seidel solver: (a) undamped problem; (b) damped problem (30\% acoustic absorption and $5 \%$ structural damping). Eigenfrequencies of the sending and receiving domains, $\Omega_{F}^{(1)}$ and $\Omega_{F}^{(2)}$, and the structure are also shown.

\subsection{Influence of particular eigenfrequencies}

The performance of the block Gauss-Seidel solver for three particular frequencies has been analysed. The example of Section 4.1 has been considered (damped situation). The aim of the analysis is to show differences in the efficiency of the method depending on the type of eigenfrequencies that are close to the excitation frequency. The studied frequencies are: i) $70 \mathrm{~Hz}$, which is close to uncoupled eigenfrequencies of the structure $(70.83 \mathrm{~Hz})$ and the receiving room $(69.27 \mathrm{~Hz})$; ii) $90 \mathrm{~Hz}$, which is not close to any of the eigenfrequencies of the problem; iii) $156 \mathrm{~Hz}$, which is close to an uncoupled eigenfrequency of the structure $(156.22 \mathrm{~Hz})$. 
Results are presented in Figure 7. As expected, the better convergence is found for case ii), which is not affected by any eigenfrequency of the problem. More iterations are required in situations $i$ ) and iii). The eigenfrequencies of the problem increase the value of the spectral radius of the iteration matrix. This phenomenon has already been predicted in the one-dimensional model presented in Section 3.2, see Equations (13) and (14).

The evolution of the relative error of the spatial averaged mean pressure $\left\langle p_{r m s}^{2}>\right.$ (the most frequently used output in sound transmission problems) is shown in Figure $7(\mathrm{~b}) . p_{r m s}=|p| / \sqrt{2}$ is the root mean square pressure that can be obtained from the pressure phasor $p$. For most of the excitation frequencies, the error is small from an engineering point of view $(<10 \%)$ after just two iterations. However, for excitation frequencies that are close to the eigenfrequencies or for undamped problems, the error is larger and more iterations are needed.

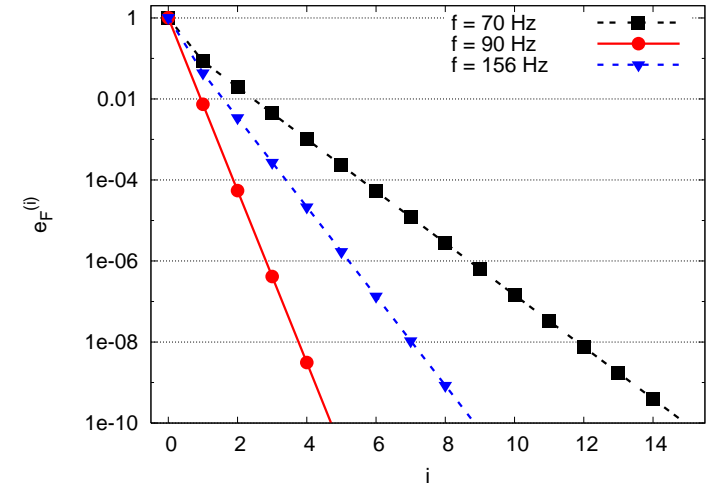

Figure 7: Behaviour of block Gauss-Seidel solver for various particular eigenfrequencies: (a) relative error in the sending domain; (b) relative error of $<p_{r m s}^{2}>$ in the receiving domain.

\subsection{Three-dimensional simulation of the sound level differ- ence}

The block Gauss-Seidel algorithm has been used for a three-dimensional analysis of sound transmission through a single lightweight wall. The room dimensions are $5.7 \times 4.7 \times 3.7 \mathrm{~m}^{3}$ (sending room) and $6.35 \times 5 \times 4 \mathrm{~m}^{3}$ (receiving room). The acoustic absorption of the rooms is $10 \%$. The dimensions of the wall are $4 \times 3 \mathrm{~m}^{2}$. It is simply supported and its geometrical and mechanical properties can be found in Table 3. The Gmsh pre- and postprocessor [28] is used for mesh generation and visualisation purposes.

In order to increase the frequency range of the analysis, a modal description of the acoustic domains is done. They are cuboids and the analytical expression of the eigenfunctions is known. With this modification, the fluid unknowns in $\mathbf{x}_{F}$ are modal contributions instead of nodal values of the pressure phasor. 
Table 3: Geometric and material properties of the lightweight wall.

\begin{tabular}{lll}
\hline Meaning & Symbol & Value \\
\hline Young's modulus & $E$ & $4.8 \cdot 10^{9} \mathrm{~N} / \mathrm{m}^{2}$ \\
Poisson's ratio & $\nu$ & 0.2 \\
Solid density & $\rho_{\text {solid }}$ & $913 \mathrm{~kg} / \mathrm{m}^{3}$ \\
Wall thickness & $\mathrm{t}$ & $0.013 \mathrm{~m}$ \\
Hysteretic damping coefficient & $\eta$ & $0.5 \%$ \\
\hline
\end{tabular}

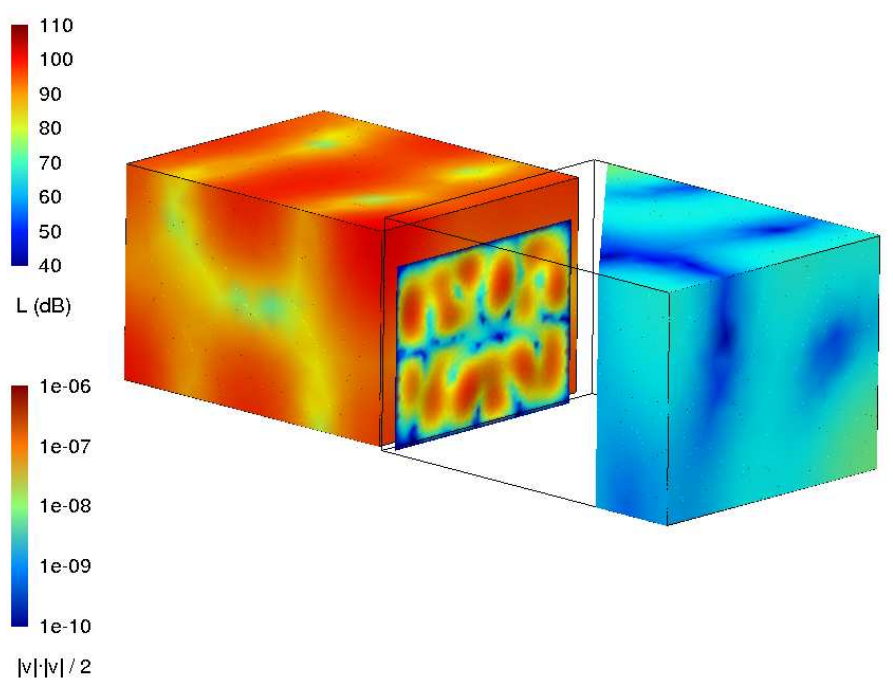

(a)

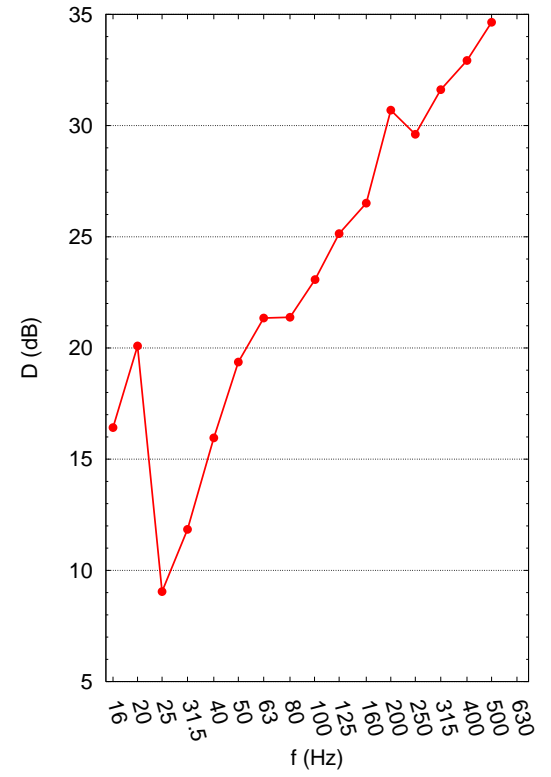

(b)

Figure 8: Sound transmission through a single wall: (a) sound pressure level in the rooms of the laboratory in $\mathrm{dB}$ and velocity field over the tested plasterboard $v_{r m s}^{2}\left(\mathrm{~m}^{2} / \mathrm{s}^{2}\right)$ for a frequency of $150 \mathrm{~Hz}$; (b) evolution of the sound level difference $D$ with frequency.

The pressure field in both rooms and the velocity distribution in the wall for a frequency of $150 \mathrm{~Hz}$ have been plotted in Figure 8(a). Note that the pressure field is measured in $\mathrm{dB}$ and the sound pressure level $L=10 \log _{10}\left(p_{r m s}^{2} / p_{0}^{2}\right)$ has been used as output $\left(p_{0}=2 \cdot 10^{-5} \mathrm{~Pa}\right.$, is a reference pressure value). $v_{r m s}$ is the root mean square velocity and can be obtained from the normal velocity phasor as $v_{r m s}=\left|v_{n}\right| / \sqrt{2}$. The sound level difference $D=L_{\text {sending }}-L_{\text {receiving, }}$, which is a measure of the insulation capacity of the wall, has been plotted in Figure 8(b). The insulation is poor at lowfrequencies. In this frequency range the calculated outputs are highly influenced by the eigenfrequencies of the problem. In the mid-frequency range the modal dependence disappears and the insulation capacity of the wall increases approximately $6 \mathrm{~dB}$ per octave which is the expected result.

The block Gauss-Seidel method behaves as described in previous sections for this 
three-dimensional problem. For those frequencies where the coupling is weak or not close to an eigenfrequency, the system of linear equations is solved with few iterations. For some particular frequencies with strong coupling more iterations are required in order to take into account the strong coupling.

\section{The case of double walls: selective coupling of fluid domains}

All the examples shown in Section 4 deal with single walls. For typical geometrical and material parameters, the acoustic domains (sending and receiving rooms) and the structure are weakly coupled, and thereby one-way coupling approaches have a good performance.

This is not the case, however, for double walls (consisting on two leaves separated by a cavity, either filled with an acoustic absorbing material or not), see Figure 9. For these applications, a one-way coupling approach or even the iterative block GaussSeidel method are not efficient or diverge. The reason is that the air cavity between walls is usually small (cavity thickness between $2 \mathrm{~cm}$ and $8 \mathrm{~cm}$ ), and thereby, $k \ell$ is also small. As shown in Section 3, this increases the stiffness of the acoustic domain and causes the divergence of the block Gauss-Seidel algorithm. To overcome these

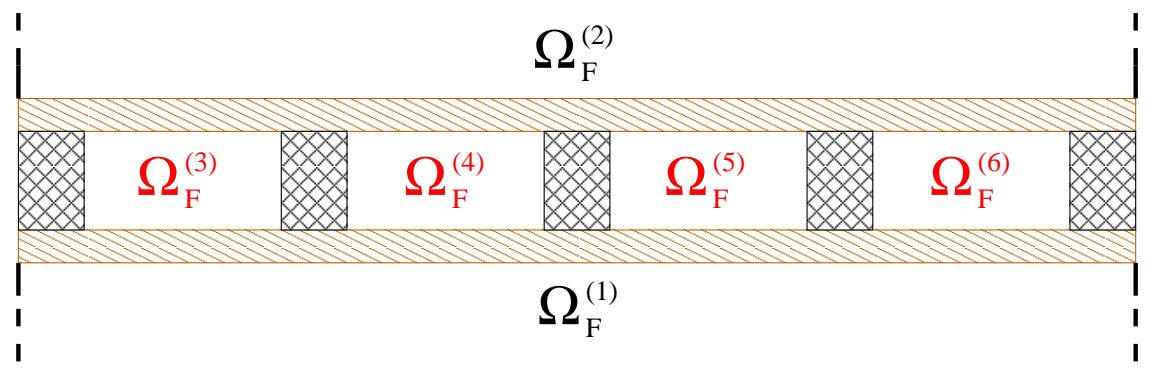

Figure 9: Sketch of a double wall. The sending and receiving rooms (1 and 2) are weakly coupled with the structure while the cavities (3, 4, 5 and 6) are strongly coupled. This information is used in the solver.

difficulties, we present here a modification of the block Gauss-Seidel algorithm. The goal is to deal with situations where some of the fluid domains are strongly coupled to the structure. The matrices in Equation (1) can be written in detail as

$$
\mathbf{F}=\left[\begin{array}{ccccc}
\mathbf{F}^{(1)} & \mathbf{0} & \cdots & \cdots & \mathbf{0} \\
\mathbf{0} & \mathbf{F}^{(2)} & \ddots & & \vdots \\
\vdots & \ddots & \ddots & \ddots & \vdots \\
\vdots & & \ddots & \ddots & \mathbf{0} \\
\mathbf{0} & \cdots & \cdots & \mathbf{0} & \mathbf{F}^{(n)}
\end{array}\right]
$$


and

$$
\mathbf{C}_{F S}=\left[\begin{array}{c}
\mathbf{C}_{F S}^{(1)} \\
\mathbf{C}_{F S}^{(2)} \\
\vdots \\
\vdots \\
\mathbf{C}_{F S}^{(n)}
\end{array}\right] \quad \mathbf{C}_{S F}=\left[\begin{array}{lllll}
\mathbf{C}_{S F}^{(1)} & \mathbf{C}_{S F}^{(2)} & \cdots & \cdots & \mathbf{C}_{S F}^{(n)}
\end{array}\right]
$$

where $n$ acoustic domains are assumed.

A selective coupling strategy will be used. The $m$ problematic fluid domains will now be solved together with the structure. They are in general the smaller fluid domains (i.e. the air cavities inside the double wall, see Figure 9), which are strongly coupled with the structure. A new matrix for the structural part of the problem including these coupled acoustic domains can be written as

$$
\mathbf{S}^{*}=\left[\begin{array}{ccccc}
\mathbf{F}^{(1)} & \mathbf{0} & \cdots & \mathbf{0} & \mathbf{C}_{F S}^{(1)} \\
\mathbf{0} & \ddots & \ddots & \vdots & \vdots \\
\vdots & \ddots & \ddots & \mathbf{0} & \vdots \\
\mathbf{0} & \cdots & \mathbf{0} & \mathbf{F}^{(m)} & \mathbf{C}_{F S}^{(m)} \\
\mathbf{C}_{S F}^{(1)} & \cdots & \cdots & \mathbf{C}_{S F}^{(m)} & \mathbf{S}
\end{array}\right]
$$

The double wall in Figure 9 is a typical situation where this selective coupling is very efficient. The sending room $\Omega_{F}^{(1)}$ and the receiving room $\Omega_{F}^{(2)}$ are weakly coupled with the structure, so the matrices describing this part of the problem are considered as independent blocks. On the contrary, the cavities between leaves (acoustic domains $\Omega_{F}^{(3)}, \Omega_{F}^{(4)}, \Omega_{F}^{(5)}$ and $\Omega_{F}^{(6)}$ ) are strongly coupled with the structure, so their related matrices are solved in the same block as the structural part of the problem, in matrix $\mathrm{S}^{*}$.

Due to this coupling, matrix $\mathbf{S}^{*}$ loses the symmetry of matrix $\mathbf{S}$, so an unsymmetric solver is required. However, the coupled acoustic domains are small and the increase in the size of the matrix is moderate. Apart from the definition of matrix $\mathbf{S}^{*}$, the rest of the iterative process remains unchanged.

\subsection{Validation: one-dimensional example}

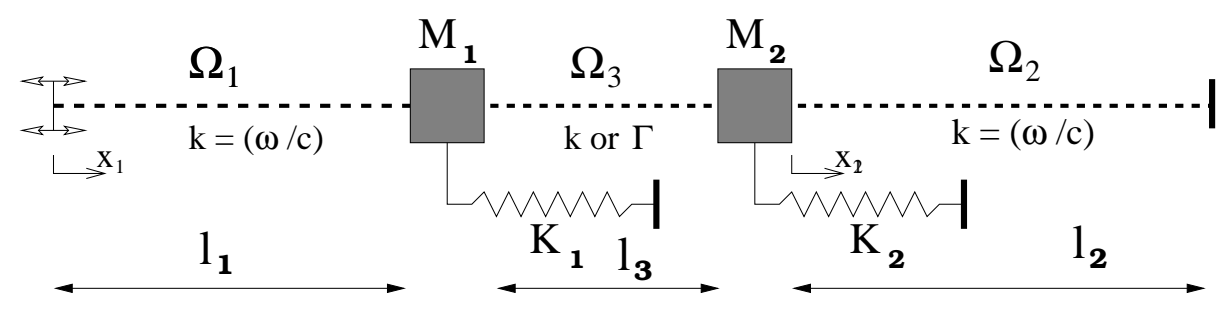

Figure 10: One-dimensional coupled system with three acoustic domains. It is used to study the performance of the selective coupling in the modelling of sound insulation of layered partitions. 
The selective coupling strategy has been used in order to solve the one-dimensional problem for layered partitions presented in [25]. An sketch of the analysed situation can be seen in Figure 10. The data in Table 4 has been considered. Note that there is no damping. The goal of this simple example is to illustrate the performance of selective coupling and compare its efficiency with the standard block Gauss-Seidel algorithm.

Table 4: Geometrical and material data for a lightweight double wall.

\begin{tabular}{lll}
\hline Meaning & Symbol & Value \\
\hline Density of leave 1 & $\rho_{\text {wall }}$ & $913 \mathrm{~kg} / \mathrm{m}^{3}$ \\
Thickness of leave 1 & $\mathrm{t}_{\text {wall }}$ & $0.013 \mathrm{~m}$ \\
Density of leave 2 & $\rho_{\text {wall }}$ & $809 \mathrm{~kg} / \mathrm{m}^{3}$ \\
Thickness of leave 2 & $\mathrm{t}_{\text {wall }}$ & $0.009 \mathrm{~m}$ \\
Length of domain 1 & $\ell_{1}$ & $3 \mathrm{~m}$ \\
Length of domain 2 & $\ell_{2}$ & $4 \mathrm{~m}$ \\
Cavity length & $\ell_{3}$ & $0.07 \mathrm{~m}$ \\
Stiffness of the single mass & $K$ & $0 \mathrm{~N} / \mathrm{m}$ \\
Structural damping & $\eta$ & $0 \%$ \\
Normal velocity & $v_{n}$ & $7.5-2.5 \cdot 10^{-3} \mathrm{i} \mathrm{m} / \mathrm{s}$ \\
Acoustic absorption & $\alpha$ & $0 \%$ \\
\hline
\end{tabular}

In Figure 11(a) the spectral radius of the iteration matrix for several values of the cavity thickness is shown. The frequency of the problem is $100 \mathrm{~Hz}$, not close to any eigenfrequency. The standard algorithm only converges for wide air cavities, whereas selective coupling converges for all the thickness range.

Figure 11(b) shows the evolution of the spectral radius with frequency for the case of a $7 \mathrm{~cm}$ thick cavity. In general terms we can see that selective coupling is convergent for all the frequency range of interest (value of the spectral radius smaller than 1) while standard block Gauss-Seidel diverges. However, both strategies have convergence problems for particular frequencies that are close to the eigenfrequencies of the undamped problem as discussed in Section 4.1.

\subsection{Application: two-dimensional example}

Selective coupling has also been used for two-dimensional problems. The example of Figure 5 is solved again, but replacing the single wall by a lightweight double wall. The material and geometrical data of the leaves can be found in Table 2. The cavity between leaves is $0.07 \mathrm{~m}$ thick. Two cases have been considered: air cavity and absorbing material (resistivity $\varrho=10^{4} \mathrm{~N} /\left(\mathrm{s} \cdot \mathrm{m}^{4}\right)$ ). The acoustic absorption is $30 \%$ and the structural damping $5 \%$.

The two larger acoustic domains have been considered as independent blocks while the acoustic cavity between leaves and the structural matrix, as well as the coupling matrices between them, have been assembled together in $\mathbf{S}^{*}$. The number of iterations required vs. the frequency has been plotted in Figure 12, for both the standard block Gauss-Seidel and the selective coupling strategies. 


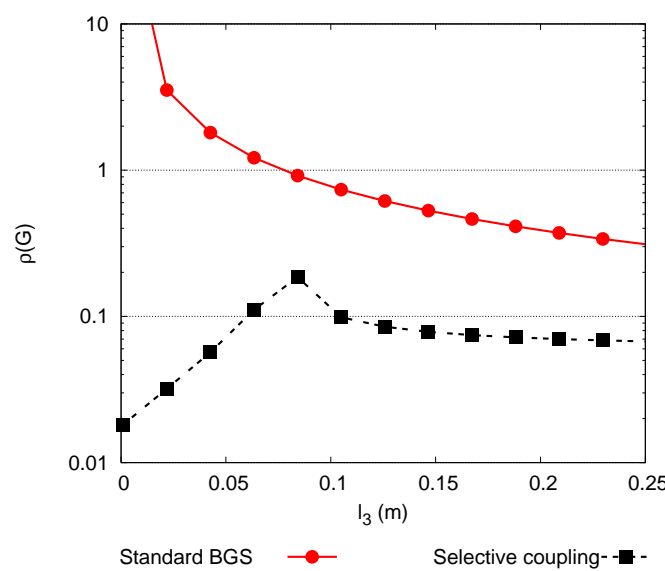

(a)

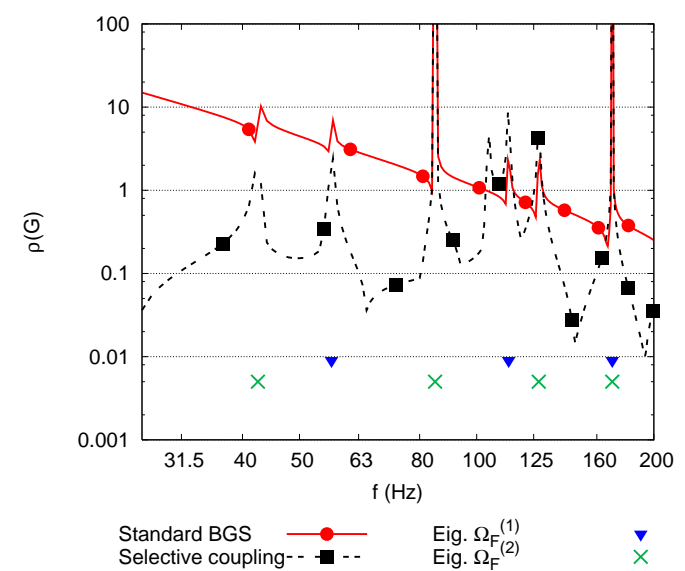

(b)

Figure 11: Selective coupling strategy applied to a one-dimensional model for a double wall. Evolution of the spectral radius of the Gauss-Seidel iteration matrix: (a) vs. cavity thickness, for a constant frequency of $100 \mathrm{~Hz}$; (b) vs. frequency, for a constant thickness of $0.07 \mathrm{~m}$.

It has to be noted that an iteration of the selective coupling strategy is computationally more expensive because of the cost of solving the linear system with matrix $\mathbf{S}^{*}$ (as compared to solving a system with matrix $\mathbf{S}$ and other small systems with matrices $\left.\mathbf{F}^{(j)}\right)$.

However, this fact is more than compensated by the better convergence behaviour of selective coupling. The convergence is quickly reached in all the frequency range; eigenfrequencies do not drastically increase the number of iterations. In fact, the only situation of non-convergence takes place for the mass-air-mass resonance of the wall. This is an eigenfrequency that couples all the subdomains of the problem (for more details, see [29]). For the studied double wall it is $91.2 \mathrm{~Hz}$. This only happens for double walls without absorbing material placed in the cavity.

As shown in Figure 12, when the problem is solved by means of a totally uncoupled procedure (i.e. standard block Gauss-Seidel), convergence is rarely reached. Sound transmission through double walls is a clear example where selective coupling is necessary.

\section{Concluding remarks}

The performance of the block Gauss-Seidel solver for interior vibroacoustic problems has been assessed both analytically and numerically. A physical interpretation of the well-known convergence condition (spectral radius of iteration matrix smaller than one) is provided. For simple one-dimensional problems, analytical expressions of the spectral radius have been obtained.

These expressions clearly reveal the negative impact of a strong coupling between the acoustic domains and the structure. Even for moderate degrees of coupling, the usual one-way coupling approach (which may be regarded as one block Gauss- 

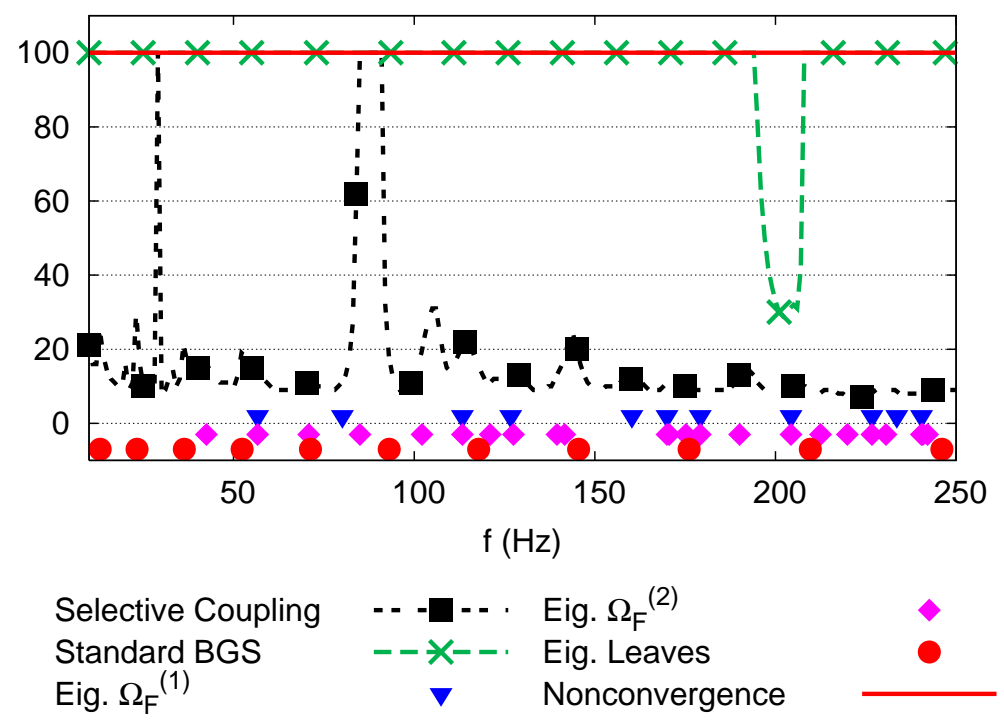

(a)
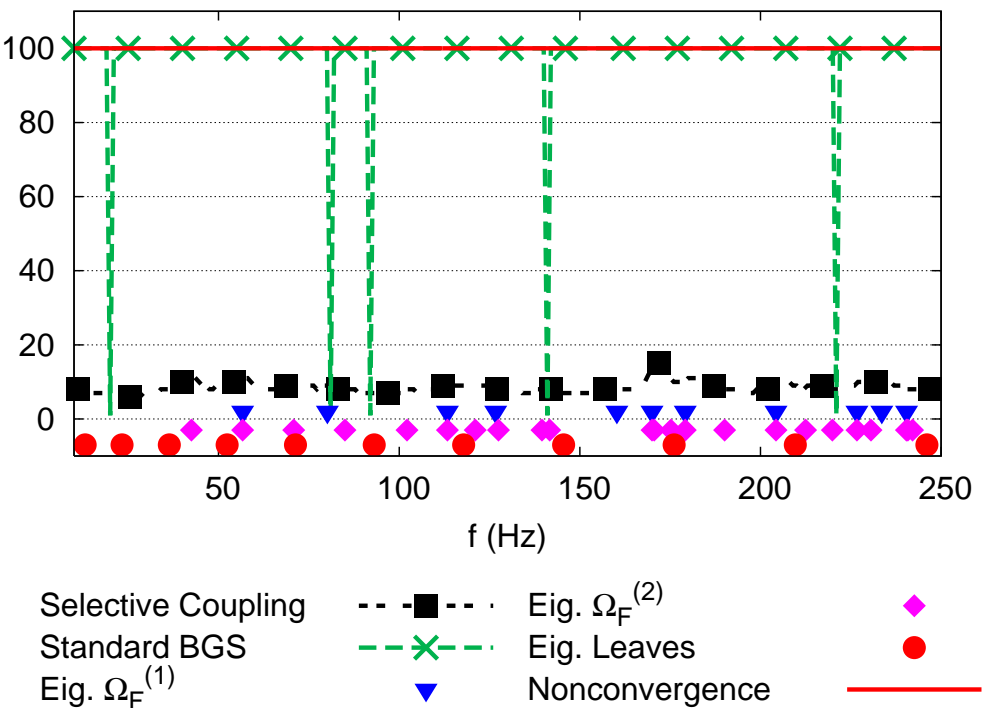

(b)

Figure 12: Application of the selective coupling to a problem of sound transmission through a lightweight double wall. Iterations required for each algorithm: (a) air cavity; (b) absorbing material. Tolerance: $=10^{-9}$; maximum number of iterations: 100 .

Seidel iteration with the appropriate ordering of blocks, along sound trajectory) is not accurate enough, and an iterative procedure to reach convergence is required.

For larger degrees of coupling, these iterations may fail to converge. This is the case, for instance, in the numerical simulation of sound transmission through double walls: the two leaves and the small acoustic cavity between them are strongly coupled.

This observation has suggested a selective coupling strategy, where the structure and the problematic acoustic domains (e.g. the cavities in a double wall) are treated 
together, in the same block.

The convergence analysis also shows the negative effect of an excitation frequency close to an (acoustic or structural) eigenfrequency for undamped problems. However, the convergence improves significantly if one uses realistic values of structural damping and acoustic absorption.

One-, two- and three-dimensional numerical examples have been used to illustrate the capabilities of the block Gauss-Seidel solver. The systematic use of this solver for the numerical simulation of sound transmission through single and double has been reported in $[25,27]$.

\section{Acknowledgements}

The financial support of the Fons Social Europeu (2003 FI 00652), Ministerio de Educación y Ciencia (BIA2007-66965, DPI2007-62395) and the Research Fund for Coal and Steel (RFSR-CT-2003-00025) is gratefully acknowledged. Free software has been used $[22,23,28,30]$.

\section{References}

[1] J.P. Morand and R. Ohayon. Fluid-structure interaction. Wiley, 1995.

[2] J. Wandinger. A symmetric Craig-Bampton method of coupled fluid-structure systems. Eng. Comput., 15(4):450-461, 1998.

[3] G.C. Everstine. Finite element formulations of structural acoustics problems. Comput. Struct., 65(3):307-321, 1997.

[4] K.J. Bathe, C. Nitikitpaiboon, and X. Wang. A mixed displacement-based finite element formulation for acoustic fluid-structure interaction. Comput. Struct., 56(2):225-237, 1995.

[5] F. Ihlenburg. Finite element analysis of acoustic scattering. Springer, 1998.

[6] P. Bouillard, V. Lacroix, and E. De Bel. A wave-oriented meshless formulation for acoustical and vibro-acoustical applications. Wave Motion, 39(4):295-305, 2004.

[7] J.P. Tuck-Lee and P.M. Pinsky. Adaptive frequency windowing for multifrequency solutions in structural acoustics based on the matrix Padé-via-Lanczos algorithm. Int. J. Numer. Methods Engng., 73(5):728-746, 2008.

[8] R. Barrett, M. Berry, T. F. Chan, J. Demmel, J. Donato, J. Dongarra, V. Eijkhout, R. Pozo, C. Romine, and H. Van der Vorst. Templates for the Solution of Linear Systems: Building Blocks for Iterative Methods, $2^{\text {nd }}$ Edition. SIAM, Philadelphia, PA, 1994.

[9] Y. Saad. Iterative methods for sparse linear systems. http://wwwusers.cs.umn.edu/ ${ }^{\sim}$ saad/books.html, 2000. 
[10] C.A. Felippa, K.C. Park, and Ch. Farhat. Partitioned analysis of coupled mechanical systems. Comput. Meth. Appl. Mech. Eng., 190(24-25):3247-3270, 2001.

[11] Q. Zhang and T. Hisada. Studies of the strong coupling and weak coupling methods in FSI analysis. Int. J. Numer. Methods Eng., 60(12):2013-2029, 2004.

[12] H.G. Matthies and J. Steindorf. Partitioned strong coupling algorithms for fluidstructure interaction. Comput. Struct., 81(8-11):805-812, 2003.

[13] S. Piperno, C. Farhat, and B. Larrouturou. Partitioned procedures for the transient solution of coupled aroelastic problems Part I: Model problem, theory and two-dimensional application. Comput. Meth. Appl. Mech. Eng., 124(1-2):79-112, 1995.

[14] S. Piperno and C. Farhat. Partitioned procedures for the transient solution of coupled aeroelastic problems - Part II: energy transfer analysis and three-dimensional applications. Comput. Meth. Appl. Mech. Eng., 190(24-25):3147-3170, 2001.

[15] C. Farhat, A. Macedo, and M. Lesoinne. A two-level domain decomposition method for the iterative solution of high frequency exterior Helmholtz problems. Numer. Math., 85(2):283-308, 2000.

[16] C. Farhat, A. Macedo, M. Lesoinne, F Roux, F. Magouls, and A. de La Bourdonnaie. Two-level domain decomposition methods with Lagrange multipliers for the fast iterative solution of acoustic scattering problems. Comput. Meth. Appl. Mech. Eng., 184(2-4):213-239, 2000.

[17] R. Tezaur, A. Macedo, and Ch. Farhat. Iterative solution of large-scale acoustic scattering problems with multiple right hand-sides by a domain decomposition method with Lagrange multipliers. Int. J. Numer. Methods Eng., 51(10):11751193, 2001.

[18] J. Mandel. An iterative substructuring method for coupled fluid-solid acoustic problems. J. Comput. Phys., 177(1):95-116, 2002.

[19] X. Feng. Interface conditions and non-overlapping domain decomposition methods for a fluid-solid interaction problem. Contemporary Mathematics, 218:417-424, 1998.

[20] P. Cummings and X. Feng. Domain decomposition methods for a system of coupled and elastic Helmholtz equations. In Ch. Lai, P.E. Bjorstad, M. Cross, and $\mathrm{O}$. Widlund, editors, $11^{\text {th }}$ International conference on domain decomposition methods (www.ddm.org), Greenwich (England), 1998.

[21] X. Feng and Z. Xie. Domain decomposition methods for a coupled vibration between an acoustic field and a plate. In T. Chan, T. Kako, H. Kawarada, and O. Pironneau, editors, $12^{\text {th }}$ International conference on domain decomposition methods (www.ddm.org), Chiba (Japan), 1999. 
[22] Satish Balay, Kris Buschelman, William D. Gropp, Dinesh Kaushik, Matthew G. Knepley, Lois Curfman McInnes, Barry F. Smith, and Hong Zhang. PETSc Web page, 2001. http://www.mcs.anl.gov/petsc.

[23] Satish Balay, Kris Buschelman, Victor Eijkhout, William D. Gropp, Dinesh Kaushik, Matthew G. Knepley, Lois Curfman McInnes, Barry F. Smith, and Hong Zhang. PETSc users manual. Technical Report ANL-95/11 - Revision 2.1.5, Argonne National Laboratory, 2004.

[24] Satish Balay, William D. Gropp, Lois Curfman McInnes, and Barry F. Smith. Efficient management of parallelism in object oriented numerical software libraries. In E. Arge, A. M. Bruaset, and H. P. Langtangen, editors, Modern Software Tools in Scientific Computing, pages 163-202. Birkhäuser Press, 1997.

[25] J. Poblet-Puig. Numerical modelling of sound transmission in lightweight structures. PhD thesis, Universitat Politècnica de Catalunya (UPC), January 2008.

[26] N. Atalla and R.J. Bernhard. Review of numerical solutions for low-frequency structural-acoustic problems. Appl. Acoust., 43(3):271-294, 1994.

[27] J. Poblet-Puig, A. Rodríguez-Ferran, C. Guigou-Carter, and M. Villot. The role of studs in the sound transmission of double walls. Acta Acust. United Acust., 95(3):555-567, 2009.

[28] C. Geuzaine and J. Remacle. Gmsh reference manual. http://www.geuz.org/gmsh/, 2009.

[29] F. Fahy. Sound and structural vibration. Academic Press, London, 1989.

[30] EDF. Code-aster home page. http://www.code-aster.org, 2009. 